\title{
LiDAR Based Vehicle Detection in Urban Environment
}

\author{
Feihu Zhang \\ Robotics and Embedded Systems \\ Technische Universität München \\ München, Germany
}

\author{
Daniel Clarke \\ fortiss $\mathrm{GmbH}$ \\ München, Germany
}

\author{
Alois Knoll \\ Robotics and Embedded Systems \\ Technische Universität München \\ München, Germany
}

\begin{abstract}
In this paper, a LiDAR based vehicle detection approach is proposed with the goal of utilizing range information. The proposed approach is based on two phases: a hypothesis generation phase to generate the potential regions and a hypothesis verification phase to recognize the corresponding vehicles. In contrast to appearance based vehicle detection systems, the proposed approach solely relies on the range information and achieves a close performance to the state-of-art. Furthermore, the proposed approach is adaptable to the environment constrains in contrast to vision based techniques, e.g. light intensity and fields of view.
\end{abstract}

Performance of the proposed approach is evaluated on a large public dataset in urban environment.

\section{INTRODUCTION}

Vehicle detection system has become an important task for advanced driver assistance systems (ADAS) in last decade. With the help of computer vision techniques, various algorithms for vehicle detection have been investigated. The workflow of vision based vehicle detection system is to segment Regions Of Interest (ROI) and classify objects within those regions [1]. Khammari et. al.utilize a gradient and Adaboost algorithm to detect vehicles based on the cameras [2]. Miller et. al. [3] and Paragios et. al. [4] have achieved their goal by applying gabor filtering and adaptive contour algorithm. In [5], vehicle detection is addressed by utilizing profile symmetry and shadows underneath a vehicle. In recent years, various features are utilized to recognize vehicles, e. g. HoG features [5], Haar-like features [6], SURF features [7] and optical flow [8]. However, such methods suffer from variations in light intensity and limited fields of view.

LiDAR is widely utilized for object detection which provides high precision range information with wide fields of view. Furthermore, LiDAR is invariant to illumination offering shape information for targets tracking [9], [10]. In [11], a LiDAR based environment perception system is proposed for autonomous vehicle. Ogawa et. al. propose an approach for pedestrian recognition using an in-vehicle LiDAR [12]. On the other hand, multi-class object recognition in static scenes are also well studied [13]. However, the challenge is that LiDAR measurements suffer from association and classification issues, in the form of points cloud data.

Integration LiDAR and cameras for object detection has attracted the attention of research communities [14], [15]. Premebida et. al. propose an approach which fuses data from LiDAR and cameras for vehicle tracking [16]. Fawzi Nashashibi et. al. combine data from LiDAR and cameras for object detection by utilizing a transferable belief model [17]. A HOG-SVM based classification system has also been proposed by Spinello et. al. to detect objects [18].

In practice, fusion based vehicle detection systems include two steps: (1) range information is first acquired by LiDAR to provide the potential objects' positions; (2) the corresponding positions are projected to the image plane for recognition with vision techniques. The above steps are combined with the name of classic LiDAR-Vision recognition system.

In our earlier work [19], we presented a vehicle detection system based on the fusion result from the camera and LiDAR. The proposed system detects potential vehicles by utilizing the cameras while the LiDAR is utilized to eliminate the false detections, in contrast to the classic LiDAR-Vision recognition system. However, the proposed approach suffers from the vision limitations since the detection is relied on the camera. Light intensity and limited fields of view often exists during the whole process.

In this paper, vehicle detection solely relies on range information for the purpose of addressing environment constrains. Our approach also consists of two phases: a hypothesis generation phase and a hypothesis verification phase. In generation phase, the Difference of Normal (DoN) operator [20] is applied to segment the 3D points cloud into the potential clusters, e. g. vehicle, pedestrian, bicycle and lamp, et. al. In verification phase, the Random Hypersurface Models (RHM) [21] is proposed to estimate the clusters' shape parameter. Furthermore, the Support Vector Machine (SVM) [22] is implemented to classify the clusters as vehicle and non-vehicle objects.

The contributions can be concluded as follows: First, a completely LiDAR based vehicle detection framework is proposed. Range information from LiDAR is solely utilized to overcome the environment constrains. Second, shape parameter for object recognition is proposed. In this paper, RHM is applied to extract potential objects' shape parameter for vehicle recognition.

The proposed approach is evaluated under real traffic scenarios provided by an off-the-shelf platform [23]. The related suites of sensors contain a Velodyne LiDAR, a stereo camera and an IMU sensor. The experiment indicates that the proposed solution achieves high reliability for vehicle detection in urban 
environment.

The remainder of this paper is structured as follows: Sec. II briefly describes the hypothesis generation phase. Sec. III introduces more details about the hypothesis verification phase. Sec. IV presents experimental results under traffic scenarios. Finally, the paper is concluded in Sec. V.

\section{HYPOTHESIS GENERATION PHASE}

In hypothesis generation phase, LiDAR points where potentially represent objects are clustered. Various methods have been developed for points cloud segmentation. However, meshing or connectivity is required for unorganized points cloud which increases the challenges [24].

Difference of Normals (DoN) operator is proposed since it directly estimated surface normal map of an unorganized points cloud [20].

\section{A. Overview on DoN}

DoN is a multi-scale operator which is approximate to the Laplacian of the Gaussian (LoG) operator [25]. It is widely used in segmentation, 3D edge detection and planar region segmentation.

\section{B. Mathematical Background}

Assuming $a$ is a point in points cloud $A$, two unit point normals $\hat{n}\left(a, r_{l}\right), \hat{n}\left(a, r_{s}\right)$ are calculated with different radii where $r_{l}>r_{s}$. Therefore, the Difference of Normals operator for point $a$ is defined as:

$$
\Delta \hat{n}\left(a, r_{s}, r_{l}\right)=\frac{\hat{n}\left(a, r_{s}\right)-\hat{n}\left(a, r_{l}\right)}{2}
$$

where $r_{s}, r_{l} \in \mathbb{R}$, and $\hat{n}(a, r)$ is the surface normal estimation at point $a$, given the support radius $r$.

Applying $\Delta \hat{n}$ to the whole points in $A$ is a vector map where a DoN operator is assigned to each point, given the radii $r_{s}$ and $r_{l}$. In addition, the operator's norm is always within the range $[0,1]$ since each DoN is the normalized sum of two unit normal vectors.

\section{Applying DoN for Segmentation}

After calculation, an important task is to consider the DoN as a salience operator to pre-filter the points cloud, with the goal of searching isolate points belong to the scale of interest. A number of objects or regions with good isolation are left within the points cloud. In this paper, Euclidean distance threshold clustering algorithm is applied to segment the results [26].

Fig. 1 illustrates the work-flow of applying DoN operator for points cloud segmentation [20]. As shown in Fig. 1, each cluster in the scenario is assigned a random color while represent as the vehicle, lamp, trees and windows, et. al.

The potentially objects are generated while the corresponding points are utilized to estimate the shape information. In this paper, only clusters on the ground plane are considered as the potential objects.

\section{HYPOTHESIS VERIFICATION PHASE}

The hypothesis verification phase is implemented with the same procedure in our previous work [19] which also consists of two steps: the parameter estimation and object classification. Shape parameters is calculated by the Random Hypersurface Model while the classification is implemented with the Support Vector Machine.

\section{A. Parameter Estimation}

\section{- Random Hypersurface Model}

In this section, Random Hypersurface Model (RHM) is utilized to calculate the shape parameter based on the range measurements. [21].

Fig. 2 illustrates that the RHM considers the measurement as a randomly scaled element of the shape boundary while the representation of the corresponding measurement is given by

$$
m_{k}+s \cdot\left(S\left(p_{k}\right)-m_{k}\right)
$$

where $s$ is considered as a random draw from the range $[0,1]$. The measurement lies on the object boundary only in condition of the scaled factor equal to 1 .

In Fig. 3, $r(\phi)$ is considered as a radial function which calculates the distance from the boundary to the center on angel $\phi$, in the form of the polar representation [27]. The mathematical model of the measurement is given by:

$$
S\left(p_{k}\right)=\left\{s \cdot r\left(\bar{b}_{k}, \phi\right) \cdot e(\phi)+m_{k} \mid \phi \in[0,2 \pi], s \in[0,1]\right\}
$$

where $e(\phi):=\left[\begin{array}{c}\cos \phi \\ \sin \phi\end{array}\right]$ is the unit vector with angle $\phi$. The radial function $r\left(\bar{b}_{k}, \phi\right)$ is the expansion of Fourier series in $\phi$. Considering $r\left(\bar{b}_{k}, \phi\right)$ as a periodic function with corresponding period $[0,2 \pi]$, the expansion of the Fourier series is given by

$$
r\left(\bar{b}_{k}, \phi\right)=a_{k}^{0}+\sum_{j=1}^{N_{F}} a_{k}^{j} \cos (j \phi)+b_{k}^{j} \sin (j \phi)
$$

where $\bar{b}_{k}=\left[a_{k}^{0}, a_{k}^{1}, b_{k}^{1}, \ldots, a_{k}^{N_{F}}, b_{k}^{N_{F}}\right]^{T}$ is the the shape parameter vector.

In Hypothesis verification phase, Fourier coefficients with lower indices represents rough shape information whereas higher indices consists of more information.

\section{- Bayesian State Estimator}

The goal of the Bayesian state estimator is to calculate the shape parameter according to the measurements on objects' surface.

In Bayesian estimation, $y_{k}$ is considered as the noisy measurement which originated from the source $z_{k}$

$$
y_{k}=z_{k}+v_{k}
$$

where $v_{k}$ is the measurement noisy which considered as the zero-mean Gaussian distribution.

Fourier descriptors are considered as the state $x_{k}$ as following 


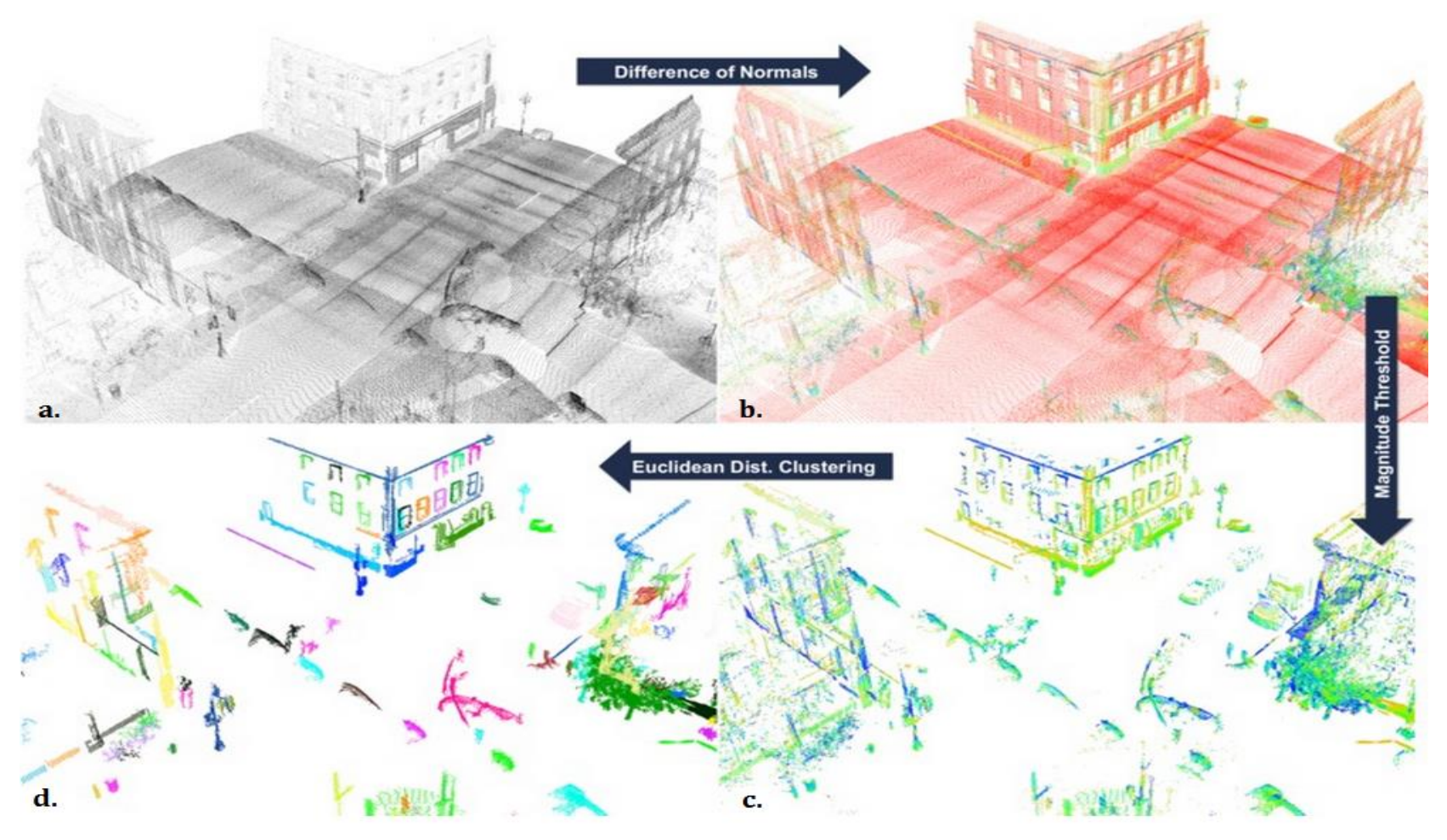

Figure 1. Overview of the pipeline in DoN segmentation [20]

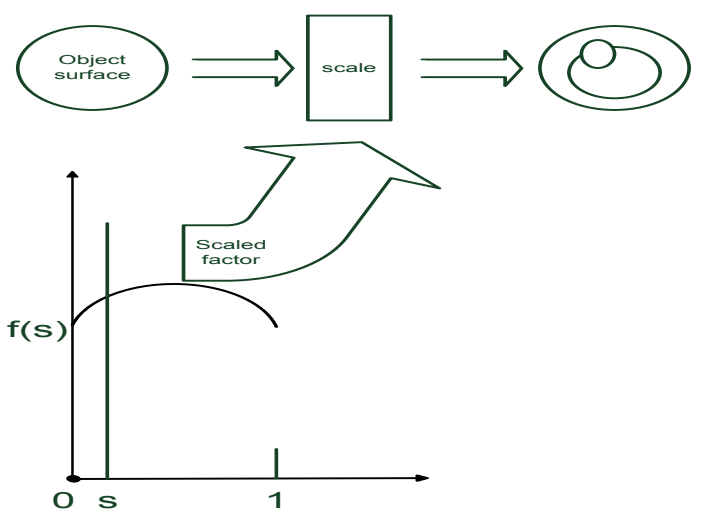

Figure 2. Random Hypersurface Model for an ellipse [19]

$$
x_{k}=A_{k} x_{k-1}+w_{k}
$$

where $A_{k}$ denotes the identity matrix for the sake of the shape parameter doesn't drift against time, $w_{k}$ is the dynamic noisy which considered as the zero-mean Gaussian distribution.

Combine (3) and (5) the closed form of the Bayesian measurement model is acquired as following

$$
\begin{aligned}
y_{k} & =z_{k}+v_{k} \\
& =s \cdot r\left(\bar{b}_{k}, \phi\right) \cdot e(\phi)+m_{k}+v_{k} \\
& :=h\left(x_{k}, v_{k}\right)
\end{aligned}
$$
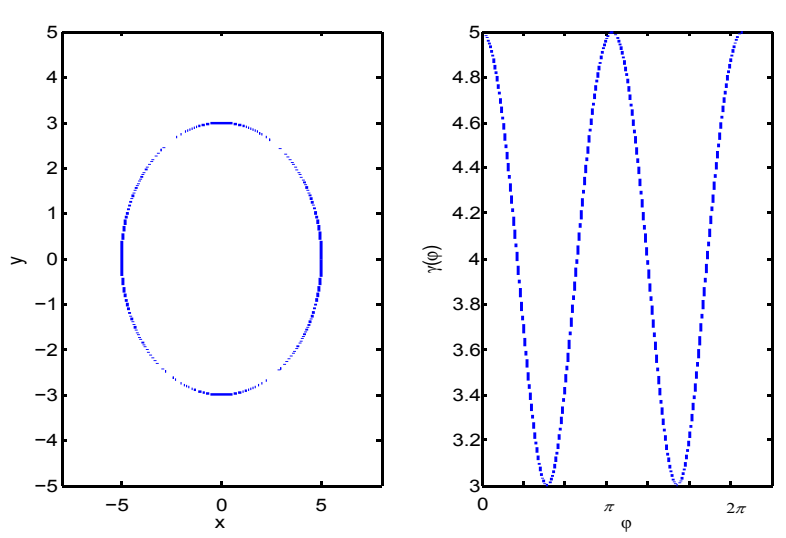

Figure 3. Example for the shape of star-convex object [19]

where the state, the measurement noise and the measurement are mapped to a non-linear condition. Finally, the Unscented Kalman filter (UKF) is utilized to estimate the corresponding states.

More details about the estimation process can be found in our previous work [19].

Fig. 4 illustrates the shape estimation result based on the RHM with a Bayesian estimator. As we can see, the Fourier coefficients represent most details of the shape information based on the measurements. The SVM is utilized to consider the corresponding parameters for vehicle and non-vehicle classification. 


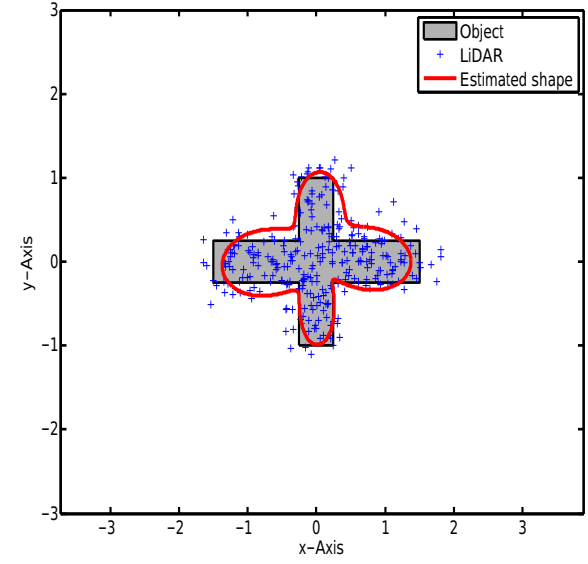

Figure 4. Example for shape estimation [19]

Table I

PERFORMANCE OF THE PROPOSED APPROACH COMPARED TO STATE-OF-ART

\begin{tabular}{|l|l|l|l|}
\hline Author & Proposed method & Y. Li [28] & L. Huang [9] \\
\hline Detection rate & $89.3 \%$ & $91 \%$ & $91.3 \%$ \\
\hline
\end{tabular}

\section{B. Object Classification}

Support Vector Machine (SVM) is proposed by Vapnik [22] which focuses on the statistical theory of learning. The general idea of the SVM is to calculate the optimal classifiers for predicting the class of new data correctly under a set of principles.

SVM has been widely utilized as a classification tool and achieved huge success in various range of applications. In this paper, the SVM is utilized as a classifier to distinguish vehicle and non-vehicle objects according to the shape parameters.

\section{EXPERIMENT EVALUATION}

In our experiment, the KITTI dataset is utilized to evaluate the performance of the proposed approach. The platform includes a large number of points cloud providing by the Velodyne LiDAR. In KITTI dataset, each frame is manually annotated 3D bounding boxes for vehicles, trucks, trams, bicycles and pedestrians. For the purpose of classification, there is a number of 5000 frames training data with 1893 objects are extracted to train the support vector (here we only considered vehicle and non-vehicle objects according to their labels, there is 2000 frames testing data for further evaluation). The successful classify rate is $91 \%$ for the training data, which illustrates that the shape parameter is a reliable vector for classification. The false classifications may cause from the orientation issue since the Fourier descriptors represent the shape well only from rear side on $2 \mathrm{D}$ coordinates.

Table I expresses the overall performance of the proposed approach compared to the state-of-art techniques, evaluated by the testing data. We have to emphasize that the proposed approach solely relies on LiDAR information comparing to the vision based algorithms. Table I clearly illustrates that the proposed approach performs a close performance to the stateof-art systems for vehicle detection. Furthermore, our approach is more adaptable to the environment constrains, e. g. light intensity and fields of view.

The contributions of the proposed approach can be concluded as follows:

First, a LiDAR based vehicle detection framework is proposed which solely relies on the range information. In our framework, LiDAR is utilized to overcome the environment constrains in contrast to the vision sensors.

Second, shape parameter for object recognition is proposed. Fourier descriptor is utilized to classify vehicle and nonvehicle objects, which is first proposed in the related domain.

The experiment indicates that the proposed approach achieves high reliability for vehicle detection compared to the state-of-art techniques in urban environments.

\section{CONCLUSION}

It is a challenge to detect vehicles from points cloud data. In previous work, LiDAR is often employed to detect objects and generate hypotheses, whereas a vision based classifier is responsible for object validation or classification. However, in this paper, a LiDAR based vehicle detection framework is proposed which solely relies on the range information. In comparison to others, the proposed scheme utilizes the object's contour information for classification by using SVM, which is first proposed in the related domain. The benefit of the proposed approach is the adaptability in complex environment constrains in contrast to vision sensors. The evaluation results illustrate that the proposed approach achieves a close performance to the state-of-art techniques, which has great potentials in future autonomous navigation systems.

\section{REFERENCES}

[1] L. Leon and R. Hirata, "Vehicle detection using mixture of deformable parts models: Static and dynamic camera," in Graphics, Patterns and Images (SIBGRAPI), 2012 25th SIBGRAPI Conference on, 2012, pp. 237-244.

[2] A. Khammari, F. Nashashibi, Y. Abramson, and C. Laurgeau, "Vehicle detection combining gradient analysis and adaboost classification," in Intelligent Transportation Systems, 2005. Proceedings. 2005 IEEE, 2005, pp. 66-71.

[3] Z. Sun, G. Bebis, and R. Miller, "On-road vehicle detection using evolutionary gabor filter optimization," Intelligent Transportation Systems, IEEE Transactions on, vol. 6, no. 2, pp. 125-137, 2005.

[4] N. Paragios and R. Deriche, "Geodesic active contours and level sets for the detection and tracking of moving objects," Pattern Analysis and Machine Intelligence, IEEE Transactions on, vol. 22, no. 3, pp. 266-280, 2000.

[5] Z. Sun, G. Bebis, and R. Miller, "Monocular precrash vehicle detection: features and classifiers," Image Processing, IEEE Transactions on, vol. 15, no. 7, pp. 2019-2034, 2006.

[6] W.-C. Chang and C.-W. Cho, "Online boosting for vehicle detection," Systems, Man, and Cybernetics, Part B: Cybernetics, IEEE Transactions on, vol. 40, no. 3, pp. 892-902, 2010.

[7] B.-F. Lin, Y.-M. Chan, L.-C. Fu, P.-Y. Hsiao, L.-A. Chuang, S.-S. Huang, and M.-F. Lo, "Integrating appearance and edge features for sedan vehicle detection in the blind-spot area," Intelligent Transportation Systems, IEEE Transactions on, vol. 13, no. 2, pp. 737-747, 2012.

[8] A. Jazayeri, H. Cai, J. Y. Zheng, and M. Tuceryan, "Vehicle detection and tracking in car video based on motion model," Intelligent Transportation Systems, IEEE Transactions on, vol. 12, no. 2, pp. 583-595, 2011. 
[9] L. Huang and M. Barth, "Tightly-coupled lidar and computer vision integration for vehicle detection," in Intelligent Vehicles Symposium, 2009 IEEE, 2009, pp. 604-609.

[10] J. Behley, K. Kersting, D. Schulz, V. Steinhage, and A. Cremers, "Learning to hash logistic regression for fast $3 \mathrm{~d}$ scan point classification," in Intelligent Robots and Systems (IROS), 2010 IEEE/RSJ International Conference on, 2010, pp. 5960-5965.

[11] R. Dominguez, E. Onieva, J. Alonso, J. Villagra, and C. Gonzalez, "Lidar based perception solution for autonomous vehicles," in Intelligent Systems Design and Applications (ISDA), 2011 11th International Conference on, 2011, pp. 790-795.

[12] T. Ogawa, H. Sakai, Y. Suzuki, K. Takagi, and K. Morikawa, "Pedestrian detection and tracking using in-vehicle lidar for automotive application," in Intelligent Vehicles Symposium (IV), 2011 IEEE, 2011, pp. 734-739.

[13] P. Espinace, T. Kollar, A. Soto, and N. Roy, "Indoor scene recognition through object detection," in Robotics and Automation (ICRA), 2010 IEEE International Conference on, 2010, pp. 1406-1413.

[14] R. Labayrade, C. Royere, D. Gruyer, and D. Aubert, "Cooperative fusion for multi-obstacles detection with use of stereovision and laser scanner," Autonomous Robots, vol. 19, pp. 117 -140, 2005.

[15] S. Rodrguez, V. Frmont, P. Bonnifait, and V. Cherfaoui, "Multi-modal object detection and localization for high integrity driving assistance," Machine Vision and Applications, vol. 14, pp. 1 -16, 2011.

[16] C. Premebida, G. Monteiro, U. Nunes, and P. Peixoto, "A lidar and vision-based approach for pedestrian and vehicle detection and tracking," in Intelligent Transportation Systems Conference, 2007. ITSC 2007. IEEE, 2007, pp. 1044-1049.

[17] F. Nashashibi, A. Khammari, and C. Laurgeau, "Vehicle recognition and tracking using a generic multisensor and multialgorithm fusion approach," International Journal of Vehicle Autonomous Systems, vol. 6, pp. 134-154, 2008 .

[18] L. Spinello and R. Siegwart, "Human detection using multimodal and multidimensional features," in Robotics and Automation, 2008. ICRA 2008. IEEE International Conference on, 2008, pp. 3264-3269.

[19] F. Zhang, D. Clarke, and A. Knoll, "Vehicle detection based on lidar and camera fusion," in Intelligent Transportation Systems, 2014. ITSC 2014. 17th International IEEE Conference on, Oct. 2014.

[20] Y. Ioannou, B. Taati, R. Harrap, and M. Greenspan, "Difference of normals as a multi-scale operator in unorganized point clouds," ArXiv e-prints, Sep. 2012.

[21] M. Baum and U. Hanebeck, "Shape tracking of extended objects and group targets with star-convex rhms," in Information Fusion (FUSION), 2011 Proceedings of the 14th International Conference on, 2011, pp. $1-8$.

[22] C. Cortes and V. Vapnik, "Support-vector networks," Machine Learning, vol. 20, pp. 273-297, 1995.

[23] A. Geiger, P. Lenz, and R. Urtasun, "Are we ready for autonomous driving? the kitti vision benchmark suite," in Conference on Computer Vision and Pattern Recognition (CVPR), 2012.

[24] A. Golovinskiy and T. Funkhouser, "Min-cut based segmentation of point clouds," in 12th International Conference on Computer Vision Workshops ICCV Workshops, 2009.

[25] H. Kong, H. Akakin, and S. Sarma, "A generalized laplacian of gaussian filter for blob detection and its applications," Cybernetics, IEEE Transactions on, vol. PP, no. 99, pp. 1-15, 2013.

[26] R. B. Rusu, "Semantic 3d object maps for everyday manipulation in human living environments," Ph.D. dissertation, Computer Science department, Technische Universitaet Muenchen, Germany, October 2009.

[27] D. Zhang and G. Lu, "Study and evaluation of different fourier methods for image retrieval," Image and Vision Computing, vol. 23, pp. 33-49, 2005.

[28] Y. Li, G. Toulminet, and A. Bensrhair, "Vehicle detection based on the stereo concept of (axis, width, disparity) symmetry map," in Intelligent Transportation Systems, 2008. ITSC 2008. 11th International IEEE Conference on, 2008, pp. 778-783. 